
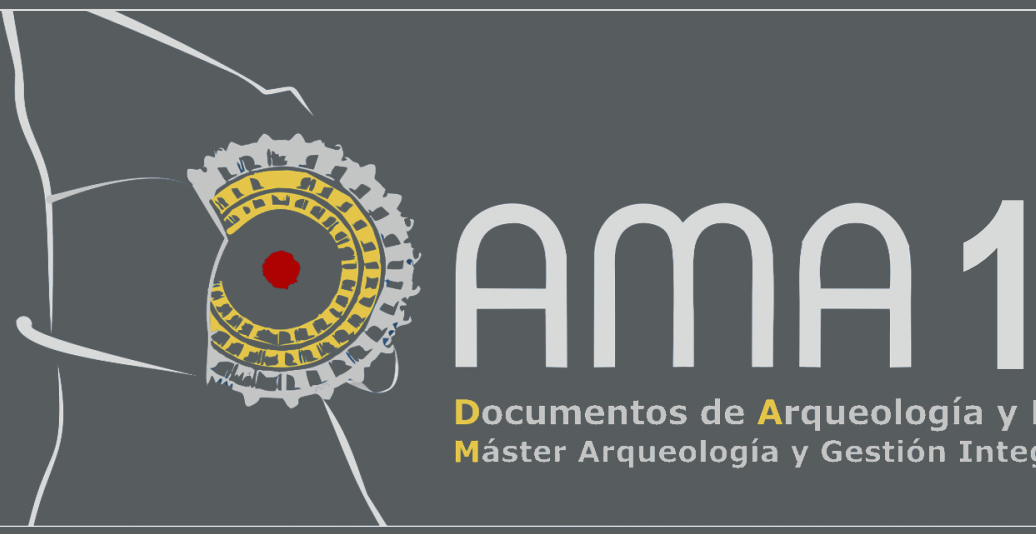

Documentos de Arqueología y Patrimonio Histórico

Máster Arqueología y Gestión Integral del Patrimonio de la Universidad de Alicante

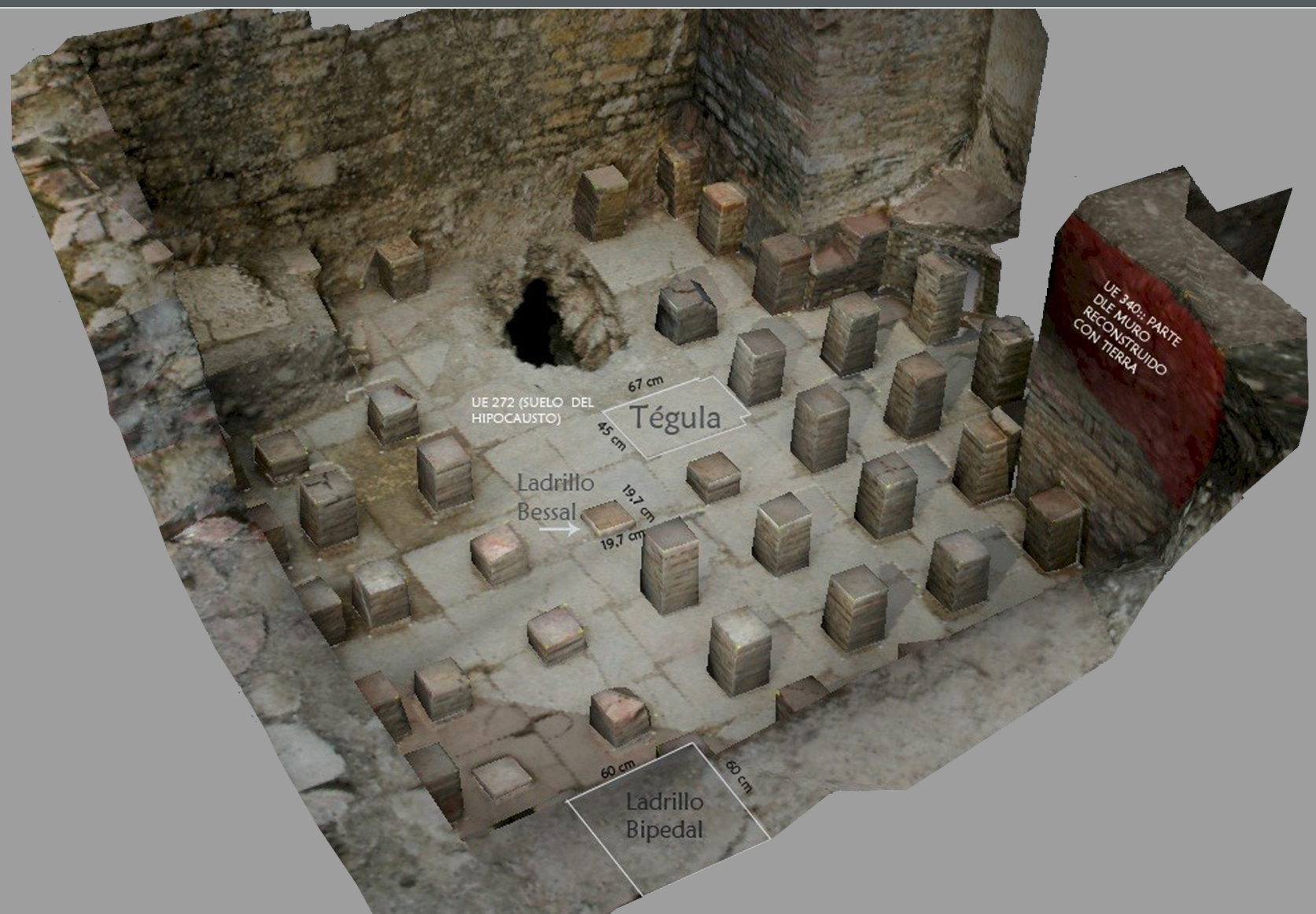

Documentos de Arqueología y Patrimonio Histórico Revista del Máster Universitario en Arqueología Profesional y Gestión integral del Patrimonio 



\section{DAMA 1}

\section{6}

DOCUMENTOS DE ARQUEOLOGÍA Y PATRIMONIO HISTÓRICO DEL MÁSTER UNIVERSITARIO EN ARQUEOLOGÍA PROFESIONAL Y GESTIÓN INTEGRAL DEL PATRIMONIO DE LA UNIVERSIDAD DE ALICANTE 


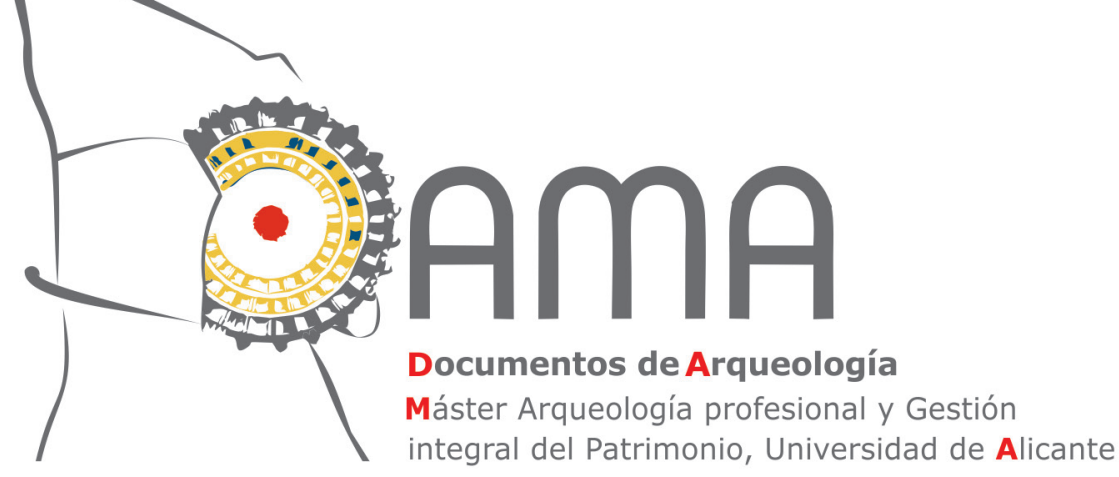

La revista electrónica DAMA. Documentos de Arqueología y Patrimonio Histórico surge como servicio para todos aquellos alumnos del Máster en Arqueología Profesional y Gestión Integral del Patrimonio de la Universidad de Alicante que se están iniciando en la investigación y cuya primera aportación a nuestra disciplina suele ser su Trabajo de Fin de Máster (TFM). Estos proyectos en muchos casos representan casi todo un curso de trabajo y esfuerzo, y con frecuencia quedan inéditos.

El objetivo de esta revista es ofrecer un medio que facilite la publicación de los resultados de sus TFM. La edición se presenta en versión digital y cuenta con su correspondiente ISSN. Se publica de forma anual en el sitio web de la Universidad de Alicante (http://web.ua.es/es/dama/) y en su repositorio (RUA). Los artículos publicados son descargables en formato PDF.

\section{Consejo de Redacción}

Directores (Coordinadores del Máster)

Carolina Doménech Belda

Fernando Prados Martínez

\section{Secretaria de Edición}

Julia Sarabia Bautista

\section{Vocales}

Los miembros de la Comisión Académica del Máster Universitario en Arqueología Profesional y Gestión del Patrimonio (http://dprha.ua.es/es/magip/comision-academica.html)

\section{Edita}

Máster Universitario en Arqueología Profesional y Gestión Integral del Patrimonio Departamento de Prehistoria, Arqueología, Historia Antigua, Filología Griega y Filología Latina

Facultad de Filosofía y Letras II

Universidad de Alicante

Ctra San Vicente del Raspeig s/n

E-03690 San Vicente del Raspeig (Alicante)

Web:http://dprha.ua.es

Teléfono: (+34) 965903663

Fax: (+34) 965903823

E-mail: revista.dama@ua.es

\section{ISSN}

$2530-2345$

\section{Portada}

Modelo fotogramétrico de un hypocaustum romano realizado por Ana Charquero 
EDITORIAL

Coordinación del máster

ENTREVISTA A SOLVEIG NORDSTRÖM: UNA MIRADA HACIA EL PASADO DE LA ESCUERA

Raúl Berenguer González ..

\section{ARQUEOLOGÍA Y MÉTODO}

EL ESTUDIO DE LOS MATERIALES CONSTRUCTIVOS DE TIERRA DEL CABEZO DEL POLOVAR (VILLENA, ALICANTE): APORTACIÓN A LAS FORMAS CONSTRUCTIVAS DE DOS PEQUEÑAS EDIFICACIONES CAMPESINAS DE LA EDAD DEL BRONCE EN EL LEVANTE PENINSULAR

María Pastor Quiles

VIVIENDAS POSTALAYÓTICAS: UNA APROXIMACIÓN A LOS ESPACIOS DOMÉSTICOS EN EL ARCHIPIÉLAGO BALEAR (550-123 ANE)

Octavio Torres Gomáriz

LAS FLOTAS DE GUARNICIÓN ROMANA EN LAS COSTAS DE HISPANIA A PARTIR DE LAS FUENTES EPIGRÁFICAS

Sergio Lledó Ramírez

UNA APROXIMACIÓN A LA RECONSTRUCCIÓN DEL PAISAJE HISTÓRICO: EL PROYECTO L'ALMISSERÀ Diana López Arroyo

NUEVAS PERSPECTIVAS PARA EL ESTUDIO ARQUEOLÓGICO DEL POBLAMIENTO RURAL MEDIEVAL EN ASPE (ALICANTE): HUERTAS Y ALQUERÍAS JUNTO AL RÍO TARAFA

Felipe Mejías López

ARQUEOLOGÍA DE LAS GUERRAS CARLISTAS

Iván Roldán Vergarachea

LA CERÁMICA DE USO ARQUITECTÓNICO EN NOVELDA: LA AZULEJERÍA DE FINALES DEL SIGLO XIX Y PRINCIPIOS DEL XX

Natalia Sala Pérez

PRÁCTICA Y USOS DE LA FOTOGRAMETRÍA DIGITAL EN ARQUEOLOGÍA

Ana $M^{a}$ Charquero Ballester.

\section{GESTIÓN Y PUESTA EN VALOR DEL PATRIMONIO}

ARQUEÓLOGOS CON DISCAPACIDAD. ARQUEOLOGÍA INCLUSIVA

Ana Samaniego Espinosa

"LES COVES DELS PESCADORS DE EL CAMPELLO": PROPUESTA DE RECUPERACIÓN, PUESTA EN VALOR Y USO PÚBLICO DE UN PATRIMONIO OLVIDADO

Ana Isabel Castro Carbonell .

LA COLONIA DE SANTA EULALIA. ESTUDIO Y PROPUESTA DE RECUPERACIÓN

Héctor de Arriba González

PROYECTO DE PUESTA EN VALOR DE LOS RESTOS DE LA GUERRA CIVIL EN LA CIUDAD DE ALICANTE

Leticia Victoria González Chouciño

ANTEPROYECTO MUSEOGRÁFICO DEL MUSEO DE HISTORIA DE SAX

Alberto Ochoa García

EL CONGRESO DE MÁSTER: UNA PROPUESTA DIDÁCTICA DEL MÁSTER DE ARQUEOLOGÍA DE LA UNIVERSIDAD DE ALICANTE

Ignasi Grau Mira, Sonia Gutiérrez Lloret, Carolina Doménech Belda, Julia Sarabia Bautista 



\title{
EL CONGRESO DE MÁSTER: UNA PROPUESTA DIDÁCTICA DEL MÁSTER DE ARQUEOLOGÍA DE LA UNIVERSIDAD DE ALICANTE
}

\author{
Ignasi Grau Mira \\ Sonia Gutiérrez Lloret \\ Carolina Doménech Belda \\ Julia Sarabia Bautista
}

En el curso 2007/08 iniciaba su andadura el Máster oficial en Arqueología profesional: herramientas para la gestión integral del Patrimonio arqueológico por la Universidad de Alicante, aprobado en 2006 (D 44/2006 de 31 de marzo, del Consell de la Generalitat, DOGV no 5233 de 4 de abril del 2006; Resolución de 22 de junio de 2006 de la Secretaría General del Consejo de Coordinación Universitaria, BOE no 157 de 3 de julio de 2006), según marco establecido en el RD 56/2005. Dicho máster fue gestado y diseñado a iniciativa del Departamento de Prehistoria, Arqueología, Historia Antigua, Filología Griega y Filología Latina de la Universidad de Alicante, como un postgrado de vocación interuniversitaria, de acuerdo a una filosofía de la que participaron también las Universidades de Jaén (con un máster similar iniciado el curso 2008-9), del País Vasco (aprobado pero no activado), y el CSIC-Universidad de Santiago, a más de instituciones como el Conjunto Arqueológico de Madīnat alZahrāy Museo de Historia de la Ciudad de Barcelona. Se concibió como un máster adaptado al Espacio Europeo de Educación Superior con un perfil investigador y profesional, orientado a la capacitación técnica y científica en Arqueología y patrimonio, la especialización de arqueólogos o la formación de especialistas en disciplinas afines vinculadas a la gestión cultural con experiencia en Arqueología.

En 2009, este máster oficial se adaptó al nuevo marco legal derivado del RD 1393/2007, teniendo en cuenta la experiencia de su praxis docente y la adaptación al diseño curricular del nuevo Grado de Historia de la Universidad de Alicante cuyo plan de estudios, de vocación generalista y con presencia equilibrada de las distintas materias y áreas de conocimiento, oferta tres itinerarios optativos en el último curso, siendo uno de ellos el de "Patrimonio cultural y divulgación de la Historia", evidenciando que ciertas necesidades superaban el marco generalista del propio grado y debían ser cubiertas por los másteres. En este sentido era necesaria una adecuación de los másteres oficiales que ya funcionaban, como era el caso. El máster modificado se puso en marcha en el curso 2010/11, ahora con la denominación de Máster oficial en Arqueología profesional y gestión integral del Patrimonio, manteniendo una de las actividades docentes más emblemáticas de la titulación: el congreso de máster. 


\section{EL CONGRESO DE MÁSTER EN EL ORIGEN}

El congreso de máster tiene su origen en la naturaleza interuniversitaria que estaba implícita en el germen del primer MásterOficial de Arqueología profesional. Con su diseño inicial se perseguía favorecer el contacto con docentes y discentes provenientes de cuatro universidades españolas y del Consejo Superior de Investigaciones Científicas, asociando especialistas de distintas líneas de investigación arqueológica. Para el óptimo aprovechamiento de esta interacción entre diferentes centros, se propuso la realización de un congreso de máster que conllevara la reunión en un mismo foro de todos los alumnos y docentes. Esta actividad se desarrollaría al terminar la fase de impartición de los contenidos teóricos del máster y en ella los alumnos participarían con breves comunicaciones que versarían sobre los contenidos teóricos recibidos, escogiendo un tema que sería posteriormente objeto de su investigación de la memoria final o trabajo de investigación tutelado (TIT). Así mismo, habría una serie de ponencias, debates y mesas redondas donde intervendrían los profesores docentes y participarían activamente los alumnos. Estos recibirían la formación necesaria para desenvolverse en este tipo de foros de debate científicos en unas sesiones preparatorias previas al congreso.

Aun cuando el proyecto interuniversitario nunca llegó a formalizarse institucionalmente por los diferentes ritmos

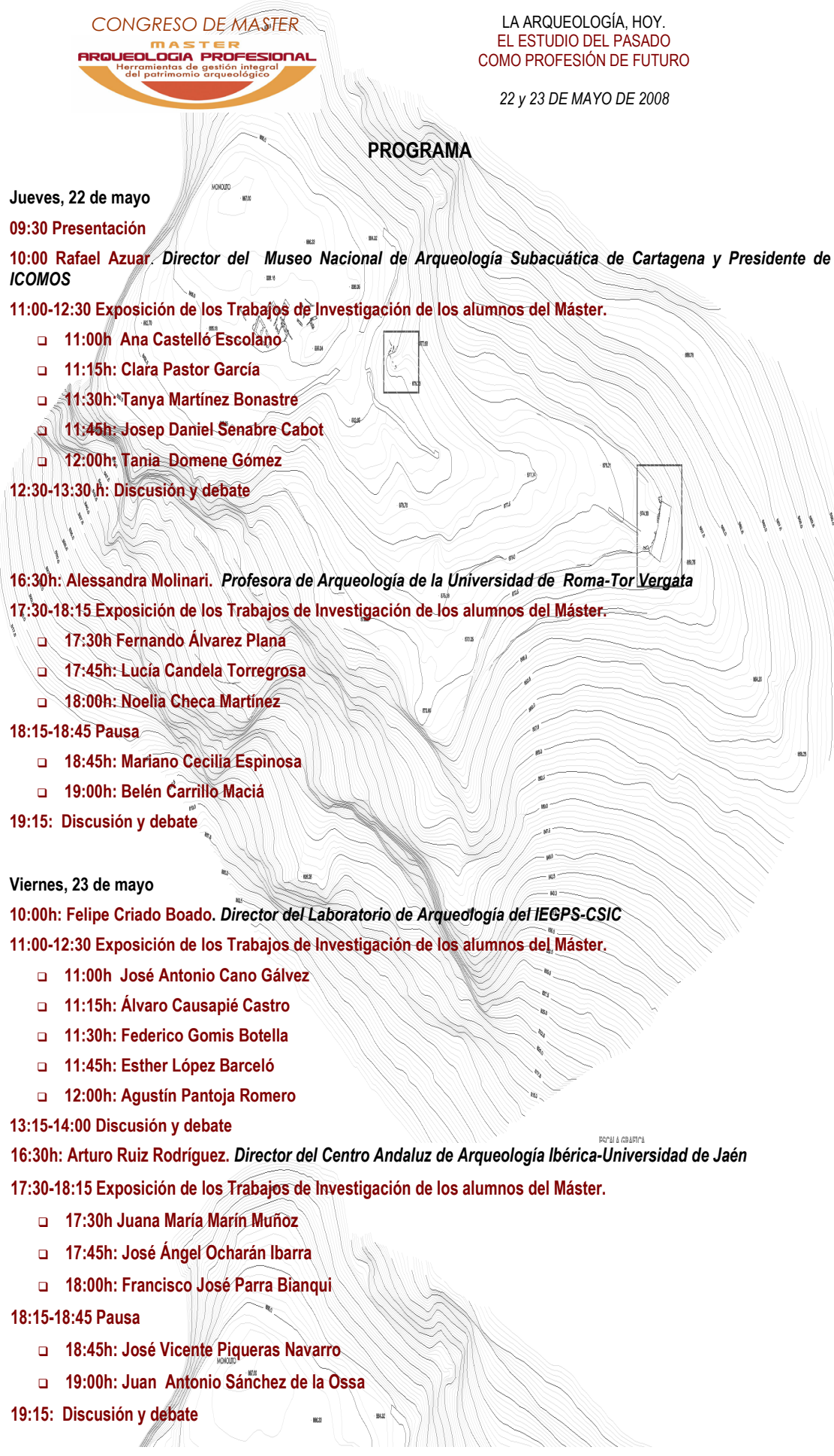

Fig. 1. Programa del I Congreso de máster que se celebró en el año 2008. 
académicos de adaptación al Espacio Europeo de Educación Superior, el congreso de máster salió adelante con el apoyo explícito de los centros promotores, cuyos miembros participaron activamente en el máster y en especial en su congreso, hasta el punto de convertirse en una de las actividades más relevantes de nuestro máster.

El primer Congreso de máster se celebró los días 22 y 23 de mayo del año 2008 bajo el lema $L a$ Arqueología hoy. El estudio del pasado como profesión de futuro (Fig.1), y continuaría durante los dos cursos siguientes en que este primer máster estuvo activo. En el último de estos tres años, en mayo de 2010, el congreso de máster adoptó un carácter internacional al dedicarse a la Arqueología Latinoamericana dentro del Marco del Proyecto Habana, vinculado a la Cooperación al Desarrollo, del Vicerrectorado de Relaciones Internacionales y Cooperación de la Universidad de Alicante. Además de ponentes nacionales, contó con la participación de profesionales venidos de la Universidad Nacional Autónoma de México, el Instituto de Antropología del Ministerio de Ciencias y Tecnología de Cuba y el Instituto de Investigaciones antropológicas de la UNAM (México) (Fig.2).

Aprovechando el marco de estudios de postgrado del Congreso del Máster de Arqueologia Profesional. Herramientas de gestión integral del Patrimonio Arqueológico, organizado por el Departamento de Prehistoria, Arqueología, Historia Antigua, Filología Griega Filologia Latina de la Universidad de Alicante, se Filogla Latina de la Universidad de Alicante, se Latinoamericana, patrocinado por el Centro de Estudios Latinoamericana, patrocinado por el Centro de Estudios
Iberoamericanos Mario Benedetti, el Proyecto La Habana Iberoamericanos Mario Benedetti, el Proyecto La Habana Cooperación y la Facultad de Filosofia y Letras.

El presente seminario tiene un doble objetivo. Por un lado, analizar el ejercicio de la arqueología en Latinoamérica en la actualidad, a partir de la presentación de los resultados obtenidos en varios proyectos de investigación desarrollados en distintos paises, asi como profundizar en las categorias y conceptos y su aplicación real desarrolladas por la denominada Arqueología Social Latinoamérica; $y$, por otro, enriquecer la formación del alumnado en general y, en particular de los participantes en el Master de Arqueología Profesional, a partir de los conocimientos y problemas existentes en ámbitos sociales y realidades arqueológicas muy diferentes de las existentes en el marco peninsular y europeo.

Con todo, este seminario también tiene el interés de servir como foro de encuentro y transmisión de experiencias de especialistas de diversos centros de investigación hispanos y latinoamericanos, con trayectorias investigadoras muy dilatadas y de gran interés cientifico.

Organiza:

Departamento de Prehistoria, Arqueologia, Historia Antigua, Filologia Griega, Filologia Latina. Universidad de Alicante. Ap. 99. E-03080 - Alicante (España)

Tf: 96903663

Coordinación:

Francisco Javier Jover Maestre y Sonia Gutiérrez Lloret

Secretaria:

Gabriel Garcia Atiénzar

Información complementaria:

javier.jover@ua.es

g.garcia@ua.es

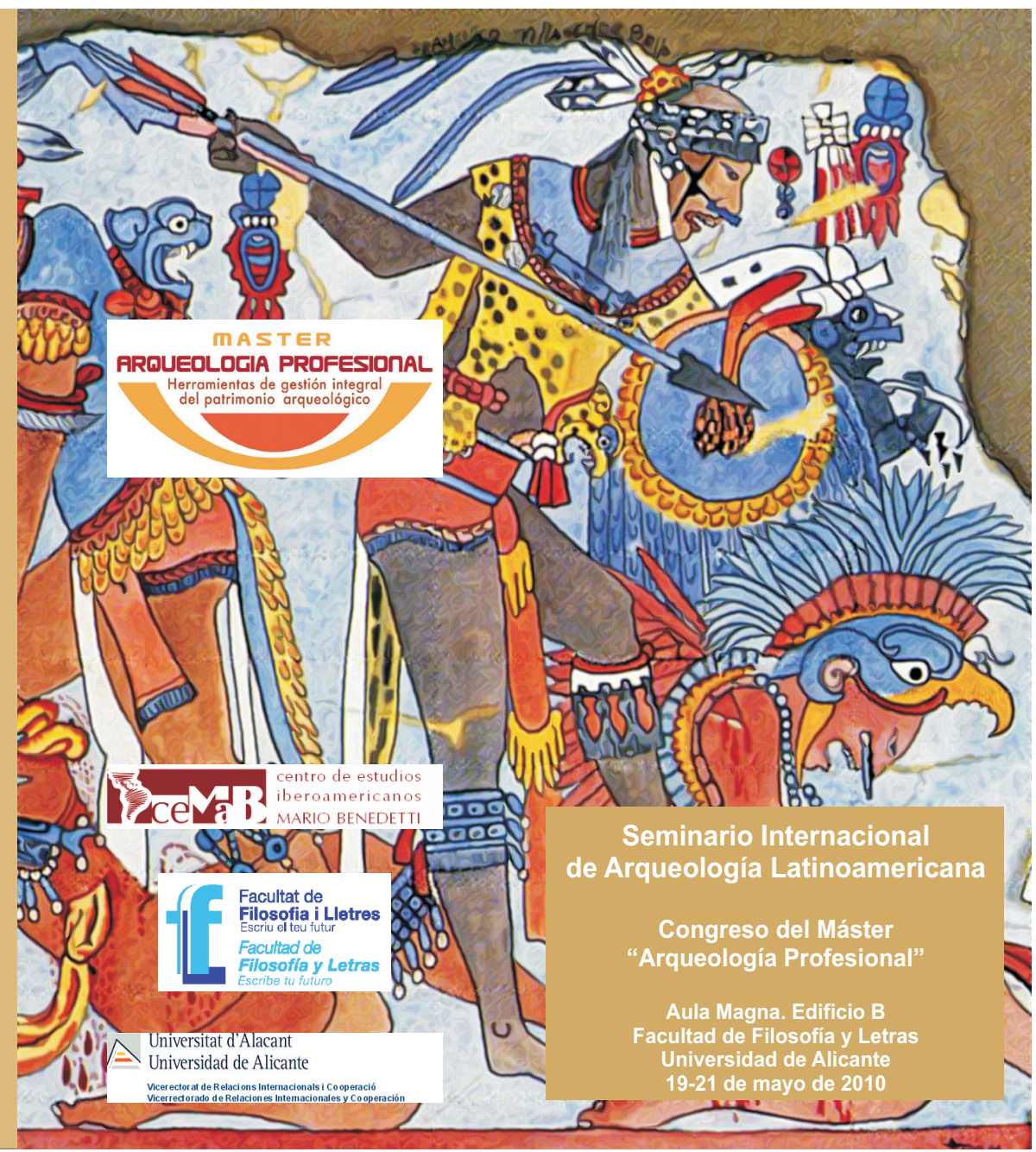

Fig. 2. Congreso de Máster 2010-Seminario Internacional de Arqueología Latinoamericana. 


\section{EL CONGRESO DE MÁSTER EN LA ACTUALIDAD}

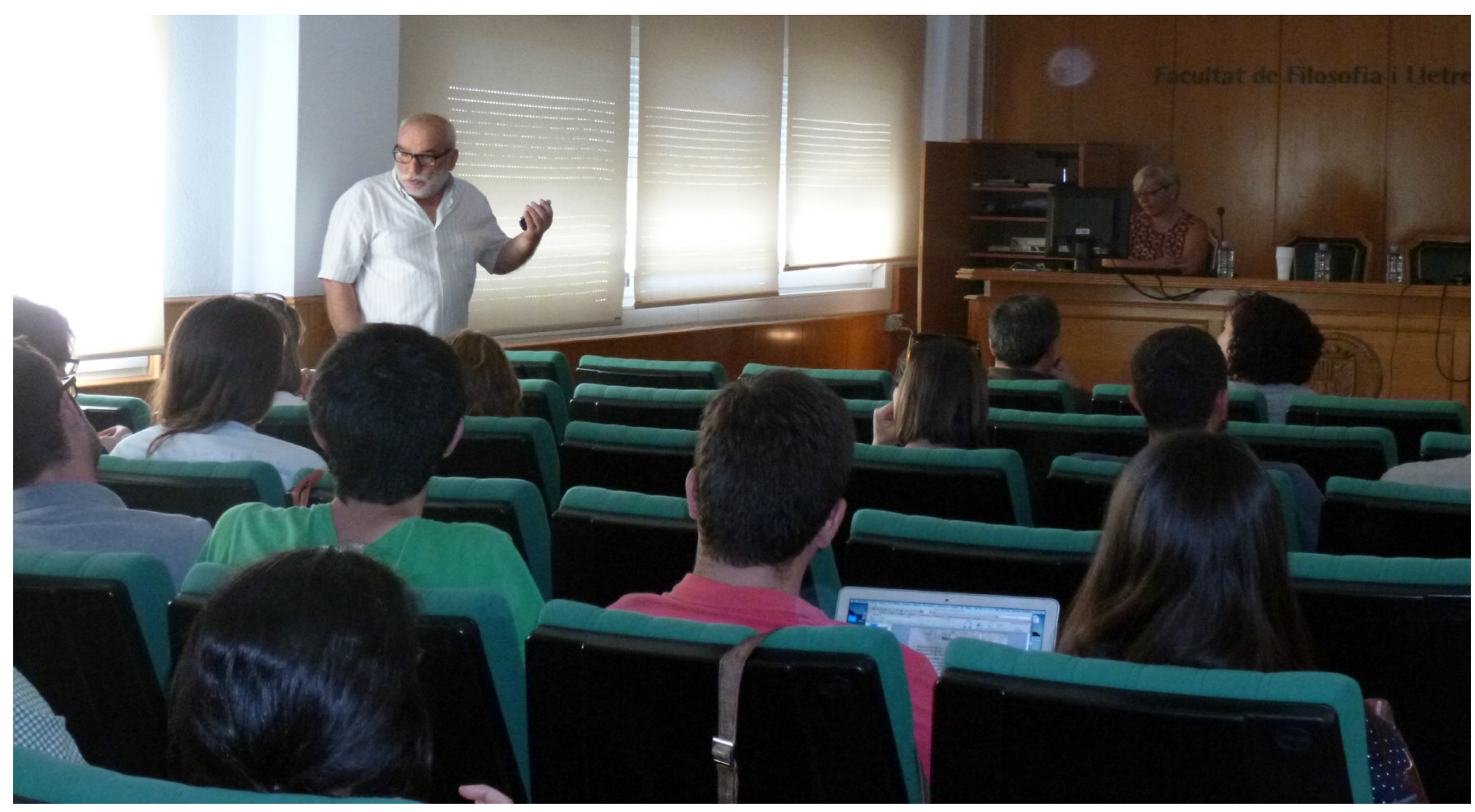

Fig. 3. El Profesor Mauro S. Hernández Pérez en un momento del debate en el Congreso de Máster de 2015.

El nuevo máster, derivado de la adaptación del anterior al marco legal que establecía el RD 1393/2007, adaptó y modificó algunos contenidos para adecuarlos a los nuevos criterios legales del perfil investigador, incluyendo una rama optativa de perfil profesional (Fig. 3).

Pero mantuvo una dilatada relación colaborativa con varias instituciones, en especial con el Instituto de Ciencias del Patrimonio (INCIPIT) del Consejo Superior de Investigaciones Científicas, el Grupo de Investigación en Patrimonio Construido (GPAC) de la Universidad del País Vasco/Euskal Herriko Unibertsitatea, el Instituto Universitario de Arqueología Ibérica y el Grupo de Investigación del Patrimonio Arqueológico de Jaén de la Universidad de Jaén y el Conjunto Arqueológico de Madīnat al-Zahrā, con quienes se inició el proyecto del máster interuniversitario que está en el origen del que ahora se imparte. Dicha relación se ha plasmado en una estrecha colaboración en las actividades de la Universidad de Alicante y en las que estos centros organizan (Máster Universitario en Restauración y Gestión Integral del Patrimonio Construido del País Vasco; Máster Universitario en Turismo, Arqueología y Naturaleza de la Universidad de Jaén, o el Curso de Postgrado. La Aplicación de las Tecnologías de Información Geográfica en Arqueología del CSIC (INCIPIT/IAM).

El congreso de máster mantiene esta vocación interuniversitaria a través de la participación, en cada edición, de dos investigadores invitados adecuados a los dos perfiles del máster: el arqueológico y el de gestión patrimonial (Fig. 4). Los ponentes invitados participan en el congreso con sendas ponencias que enmarcan los diferentes proyectos de investigación que el alumnado expone como base conceptual de su proyecto de Trabajo Fin de Máster (TFM). 


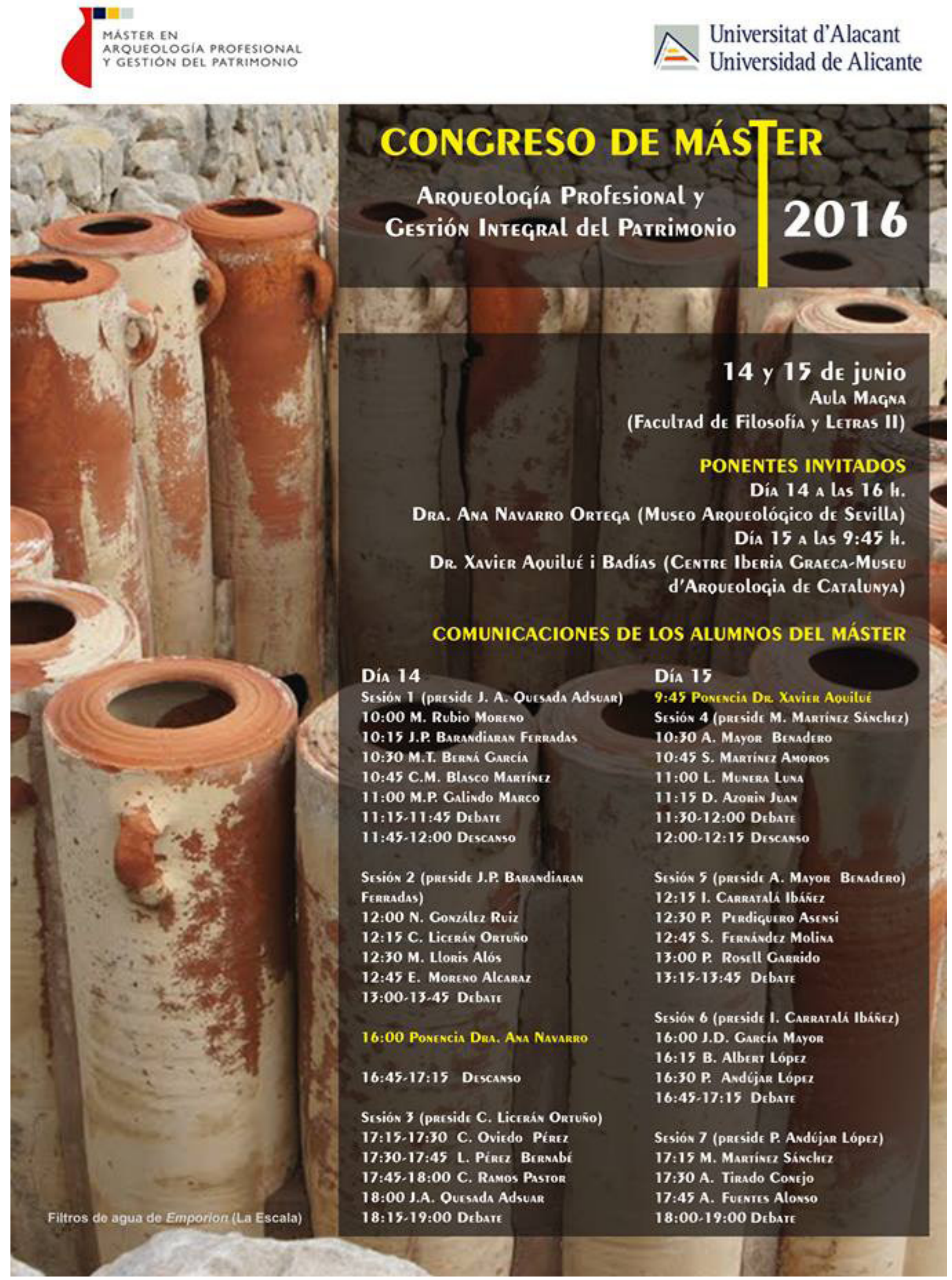

Fig. 4. Cartel del último Congreso de Máster, celebrado en junio de 2016. 
En la actualidad el congreso de máster configura una asignatura de 2 créditos obligatorios de la estructura del máster y consta de dos componentes principales. Una primera sesión preparatoria en las que se organizan el orden de participación, la presidencia de las mesas y la moderación de los debates. Así mismo se define el esquema de las presentaciones para que se ajusten a un formato lo más completo posible de lo que debe contener una propuesta de un proyecto de investigación o de puesta en valor. El congreso de máster propiamente dicho, consta de las sesiones desarrolladas por lo general en dos días, en que los alumnos se enfrentan a la presentación pública de un proyecto, ensayando los parámetros que rigen la solicitud de proyectos competitivos y la asistencia a un congreso. Las propuestas presentadas se someten a un debate y discusión en la que intervienen los alumnos del curso, el claustro de profesorado propio del máster y los profesores invitados, con la finalidad de precisar, aclarar y refinar algunos de los aspectos de los proyectos presentados.

Durante estos años han participado en el máster numerosos investigadores nacionales e internacionales, que han contribuido a su éxito. Entre ellos Arturo Ruiz (Instituto de Arqueología Ibérica), Vicente Salvatierra (Universidad de Jaén), Felipe Criado (INCIPIT), Rafael Azuar (Museo Nacional de Arqueología Subacuática-ICOMOS), Alessandra Molinari (Università di Roma-Tor Vergata), Amelia Rodríguez (Universidad de Las Palmas de Gran Canaria), Agustín Azkarate (Universidad del País Vasco), Xavier Aquilué (Centre Iberia-Graeca-Museu d'Arqueologia de Catalunya), Ana Navarro (Museo Arqueológico de Sevilla), Patrice Cressier (Université Lyon, CIHAM-UMR 5648), Antonio Vallejo (Conjunto Arqueológico de Madīnat al-Zahrā), César Parcero (CSIC), Camila Gianotti (Universidad de la República- Uruguay), Jesús Carlos Lazcano (Universidad Nacional Autónoma de México), Racso Fernández (Instituto de Antropología-Cuba), Armando Rangel (Universidad de la Habana), Carlos Arredondo (Colegio San Gerónimo de La Habana), Vicente Lull y Rafael Micó (Universitat Autónoma de Barcelona), Joan Seguí (Museu Valencià d'Etnologia), Julia Beltrán (Museu d'Història de la Ciutat de Barcelona), Gonzalo Ruiz Zapatero (Universidad Complutense de Madrid), Thomas Schattner (DAI de Madrid), Bernat Martí (SIP de Valencia).

\begin{tabular}{|l|l|l|}
\hline \multicolumn{1}{|c|}{ Asignaturas } & \multicolumn{1}{|c|}{ Descriptor } & \multicolumn{1}{|c|}{ Contenido } \\
\hline $\begin{array}{l}\text { INTER - MODELOS } \\
\text { 39811- CONGRESO } \\
\text { DE MASTER }\end{array}$ & $\begin{array}{l}\text { BLOQUE TEMÁTICO 4.- Diseño } \\
\text { de un proyecto de investigación } \\
\text { Marster en una reunión científica } \\
\text { con ponencias del profesorado } \\
\text { especialista, mesas redondas y } \\
\text { presentación de comunicaciones } \\
\text { con las propuestas de los trabajos } \\
\text { de investigación del alumnado. }\end{array}$ & $\begin{array}{l}\text { Disposición en presentación pública ante } \\
\text { alumnos y profesores invitados el proyecto } \\
\text { atendiendo a los siguientes parámetros: } \\
\text { objetivos, tema, caracterización cultural y } \\
\text { cronológica, planteamientos teóricos, } \\
\text { metodología, fases (plan de trabajo), resultados } \\
\text { esperados y significación para el avance del } \\
\text { conocimiento(Caracterizar el objeto de estudio). } \\
\text { Formato congreso: mesas con presidente y } \\
\text { debate. } \\
\text { Modalidad: ppt de 15 pantallas. }\end{array}$ \\
\hline $\begin{array}{l}\text { 39812- TRABAJO FIN } \\
\text { DE MASTER }\end{array}$ & $\begin{array}{l}\text { Asignatura dedicada a la realización } \\
\text { de trabajos tutelados de un proyecto } \\
\text { integrador de la globalidad del } \\
\text { curso, propuesto por los alumnos o } \\
\text { elegido por éstos entre una lista de } \\
\text { propuestas previamente conocida. }\end{array}$ & $\begin{array}{l}\text { Exposición de los resultados en acto público } \\
\text { ante un tribunal evaluador }\end{array}$ \\
\hline
\end{tabular}

Fig. 5. Asignaturas y lecciones vinculadas al proyecto investigador. 
Cabe reseñar que el congreso de máster forma parte de un proceso de progresión paulatina en relación a otras dos asignaturas vinculadas al diseño investigador distribuidas una al inicio del curso, dedicada a proporcionar las herramientas básicas para el diseño y planificación de un proyecto investigador, y otra, la del Trabajo Fin de Máster, que desarrolla y culmina el proyecto (Fig. 5). De esta manera, el congreso del máster se integra plenamente en el diseño curricular de la titulación, y ha devenido en una de las actividades de mayor aceptación entre el alumnado y los profesores invitados.

\section{ANEXO 1. IMÁGENES DE ALGUNOS DE LOS CONGRESOS DE MÁSTER MÁS RECIENTES}

\section{Congreso de Máster 2014}
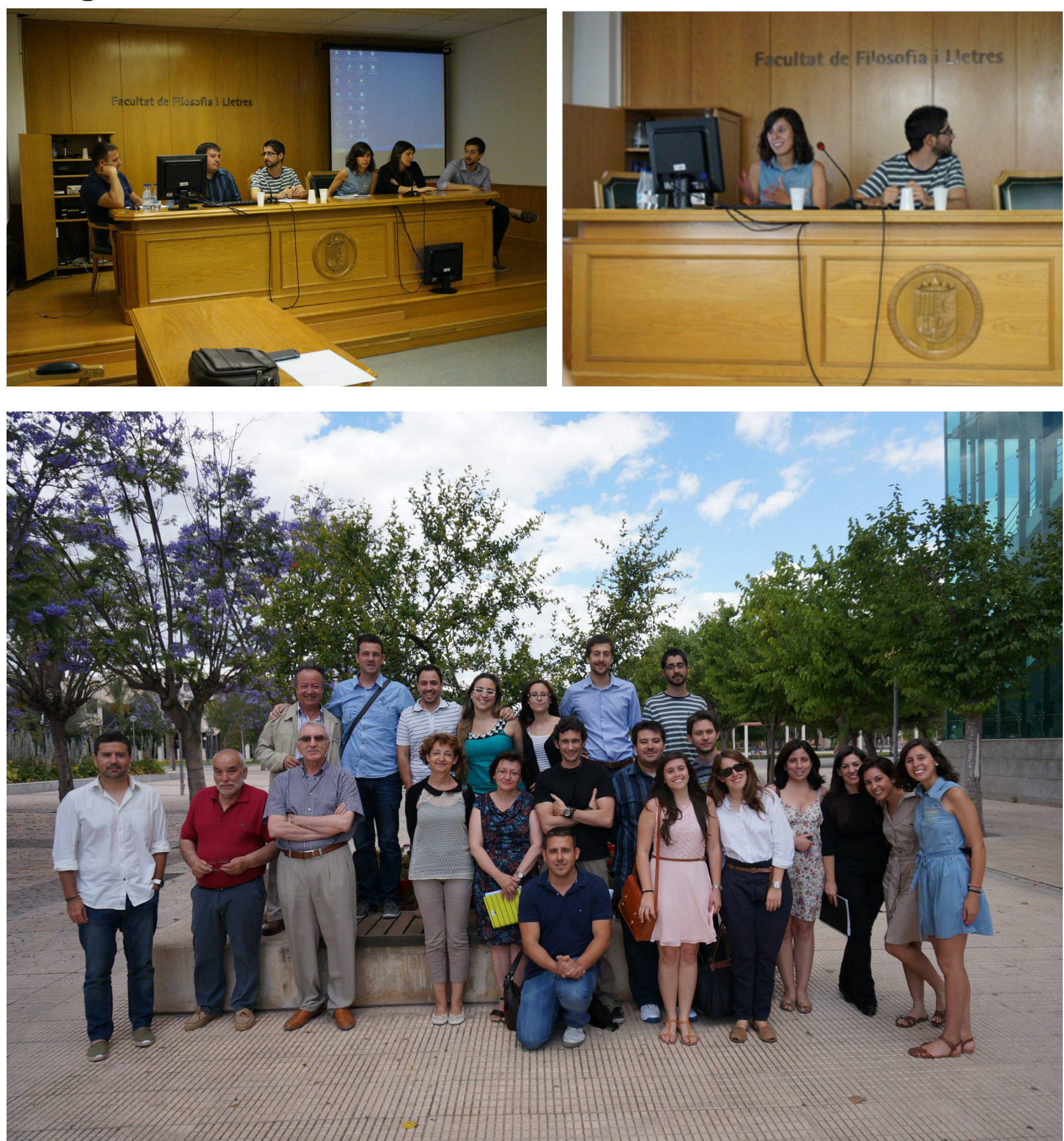


\section{Congreso de Máster 2015}
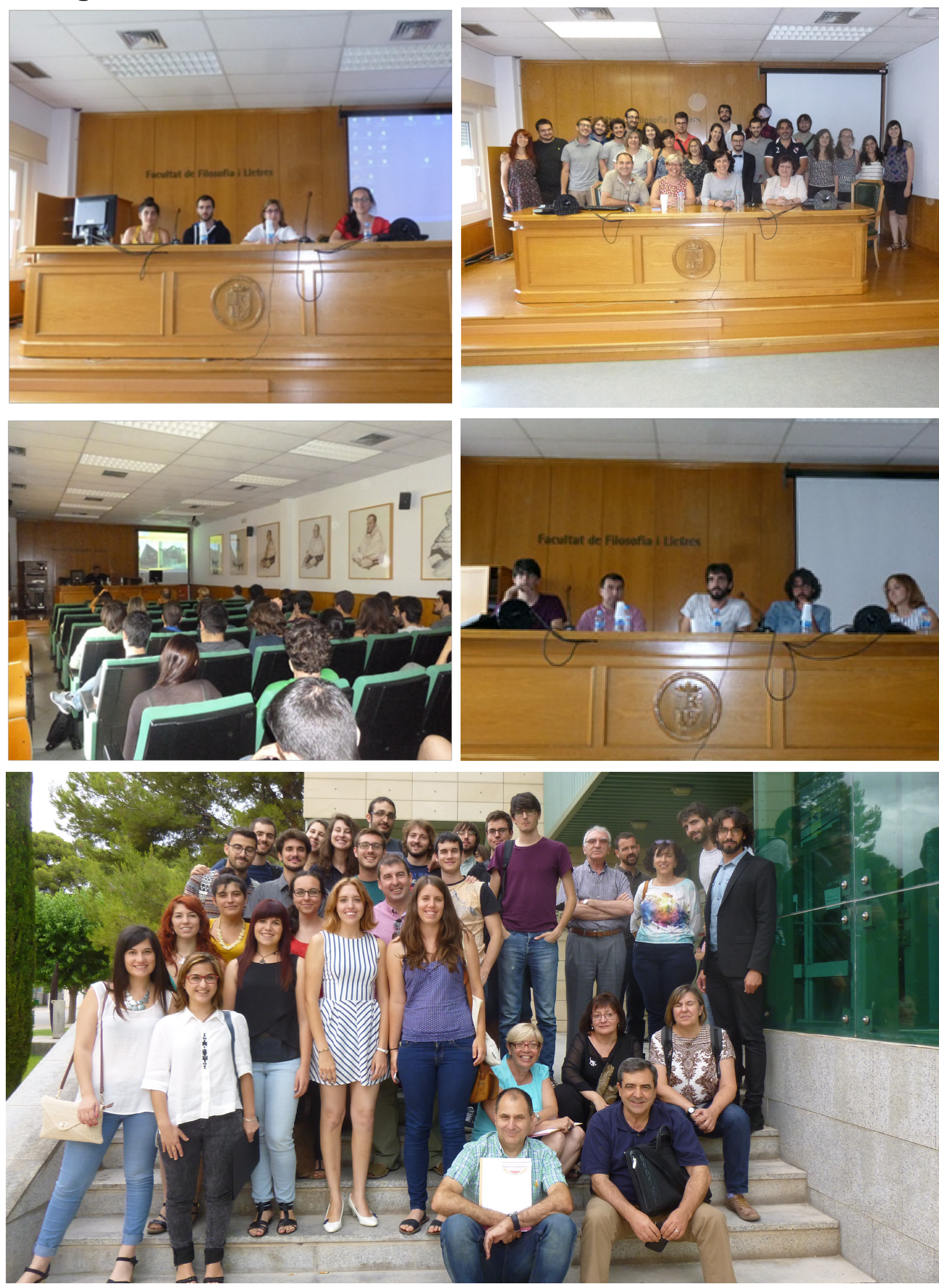


\section{Congreso de Máster 2016}
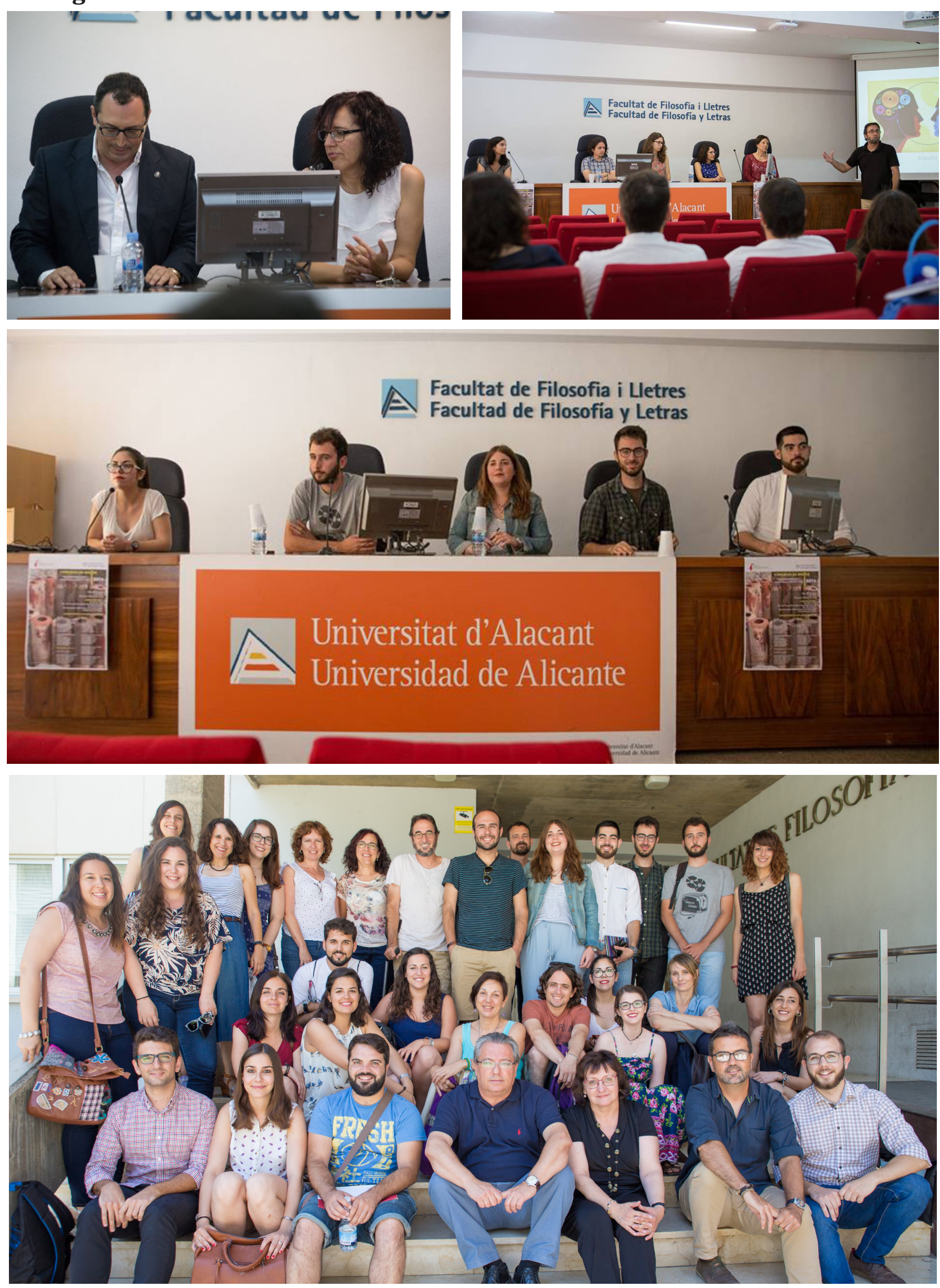


\section{ANEXO II: PROYECTOS DE LOS ALUMNOS PRESENTADOS A LOS CONGRESOS}

\begin{tabular}{|c|c|}
\hline & I Congreso de Máster \\
\hline Trabajos de In & vestigación Tutelados (TIT) curso 2007-08 \\
\hline Alumno/a & Contenido \\
\hline Fernando Álvarez Plana & $\begin{array}{l}\text { La práctica de la Arqueología de la Arquitectura desde su } \\
\text { origen hasta la actualidad }\end{array}$ \\
\hline Lucía Candela Torregrosa & $\begin{array}{l}\text { Primera aproximación a los materiales islámicos del } \\
\text { Castellar de Morera (Elche, Alicante) }\end{array}$ \\
\hline José Antonio Cano Gálvez & Repertorios domésticos de El Puig d'Alcoi \\
\hline María Belén Carrillo Maciá & Arqueología urbana en la ciudad de Elche (Alicante) \\
\hline Ana Castelló Escolano & La cerámica de Alcora \\
\hline Álvaro Causapié Castro & Arqueología funeraria romana en la provincia de León \\
\hline Mariano Cecilia Espinosa & Arqueología de la Arquitectura en Orihuela (Alicante) \\
\hline Noelia Checa Martínez & $\begin{array}{l}\text { Ánforas tardorromanas. Primera aproximación al estudio } \\
\text { de materiales cerámicos de la prospección del Castellar de la } \\
\text { Morera (Elche, Alicante) }\end{array}$ \\
\hline Tania Domene Gómez & $\begin{array}{l}\text { Calle Quevedo } n^{\circ} 2 \text {, Valencia: un estudio parcial de sus restos } \\
\text { materiales }\end{array}$ \\
\hline Federico Gomis Botella & Sistemas de registro en Arqueología \\
\hline Esther López Barceló & $\begin{array}{l}\text { Los enterramientos romanos de La Picola (Santa Pola, } \\
\text { Alicante). Estado de la cuestión }\end{array}$ \\
\hline Juana María Marín Muñoz & Molinos de papel. El Calcolítico en Caravaca (Murcia) \\
\hline Tanya Martínez Bonastre & $\begin{array}{l}\text { Guía didáctica del Parque Arqueológico Tolmo de Minateda } \\
\text { (Hellín, Albacete) }\end{array}$ \\
\hline Cristina Meseguer Ibánez & Arqueología y gestión del patrimonio \\
\hline José Ángel Ocharán Ibarra & El santuario rupestre ibérico de La Nariz (Moratalla, Murcia) \\
\hline Agustín Pantoja Romero & El acueducto "dels Enamorats" (Tibi, Alicante) \\
\hline Francisco José Parra Bianqui & $\begin{array}{l}\text { Cultos prerromanos en la Península Ibérica a través de la } \\
\text { Epigrafía }\end{array}$ \\
\hline Clara Pastor García & Proyecto de exposición: la cerámica verde-manganeso califal \\
\hline José Vicente Piqueras Navarro & $\begin{array}{l}\text { Historiografía de las necrópolis ibéricas de la provincia de } \\
\text { Albacete }\end{array}$ \\
\hline $\begin{array}{l}\text { Juan Antonio Sánchez de la } \\
\text { Ossa }\end{array}$ & Historia de un vaso cerámico \\
\hline Josep Daniel Senabre Cabot & $\begin{array}{l}\text { Propuesta de puesta en valor del patrimonio de Relleu } \\
\text { (Alicante) }\end{array}$ \\
\hline
\end{tabular}




\section{Congreso de Máster}

Trabajos de Investigación Tutelados (TIT) curso 2008-09

\section{Alumno/a}

Contenido

\begin{tabular}{|c|c|}
\hline Laura Acosta Pradillos & El Bronce final en las comarcas meridionales valencianas \\
\hline Rubén Albero Molina & Las masías de Polop Alt (Alcoy). Un paisaje contemporáneo \\
\hline Nerea Bernabeu Sepulcre & La presencia de materiales egipcios en yacimientos alicantinos \\
\hline Juan Antonio Bravo Sáez & Vía Augusta \\
\hline Patricio Domene Prats & $\begin{array}{l}\text { Los elementos de molienda en el Cabezo Rodondo (Villena, } \\
\text { Alicante) }\end{array}$ \\
\hline Magdalena Gomar Miñana & $\begin{array}{l}\text { El papel de la Arqueología en la recuperación de la Memoria } \\
\text { Histórica }\end{array}$ \\
\hline Carlos Jaén Martínez & El Estado de la Arqueología en la Educación Secundaria \\
\hline $\begin{array}{l}\text { Debora Marcela Kiss de } \\
\text { Arrendondo }\end{array}$ & $\begin{array}{l}\text { El castillo de Benifallim, Alicante. Una aproximación desde la } \\
\text { arqueología }\end{array}$ \\
\hline Jorge Alberto López Lillo & $\begin{array}{l}\text { Estructuras de combustión en el entorno de la sierra de } \\
\text { Fontcalent (Alacant): un primer acercamiento a su estudio }\end{array}$ \\
\hline María Esther Maciá Sánchez & Conservación preventiva de materiales orgánicos \\
\hline Ximo Martorell Briz & $\begin{array}{l}\text { Arte y territorio en el Río Grande (La Canal de Navarrés, } \\
\text { Valencia) }\end{array}$ \\
\hline Daniel Mateo Corredor & $\begin{array}{l}\text { Las relaciones comerciales entre Hispania Ulterior e Italia en } \\
\text { época imperialista (ss. III a.C.- II d.C.): Metodología e hipótesis } \\
\text { de una investigación }\end{array}$ \\
\hline David Nadal Barberá & $\begin{array}{l}\text { Estudio del culto en la religión ibérica vinculado a las aguas } \\
\text { en la Península Ibérica desde el s. VII al I a.C. }\end{array}$ \\
\hline José Carlos Pardines Castillo & $\begin{array}{l}\text { Aplicación de los SIGs al estudio del paisaje de la Foya de } \\
\text { Castalla (Alicante) }\end{array}$ \\
\hline Francisco Raya Ortiz & $\begin{array}{l}\text { La necrópolis del Mas del Regall, Alcoy. Nuevos datos sobre el } \\
\text { proceso orientalizante en la Comarca de l'Alcoià-El Contat }\end{array}$ \\
\hline María Lourdes Rebollo Padial & Técnicas de construcción en la villa romana de Rufio (Italia) \\
\hline Patricio Ruiz Adsuar & Ilš a la luz de los datos arqueológicos \\
\hline Samuel Serrano Salar & Las vajillas idílicas \\
\hline Cristina Tamayo Pérez & $\begin{array}{l}\text { Caracterización de las unidades de asentamiento de la Edad } \\
\text { del Bronce en el Sureste de la Península Ibérica }\end{array}$ \\
\hline
\end{tabular}




\begin{tabular}{|c|c|}
\hline \multicolumn{2}{|c|}{ Trabajos de Investigación Tutelados (TIT) curso 2009-10 } \\
\hline Alumno/a & Contenido \\
\hline Paula Bernabeu Sanz & Los bronces romanos del Museo de Villajoyosa (Alicante) \\
\hline Oscar Blanco Fernández & $\begin{array}{l}\text { Terrabona (Tous, Valencia): de alquería a despoblado. } \\
\text { Análisis preliminar de un despoblado a orillas del Júcar }\end{array}$ \\
\hline Fernando Cañizares Navarro & $\begin{array}{l}\text { El ciclo del vino en Villena y la colección etnográfica Jerónimo } \\
\text { Ferriz }\end{array}$ \\
\hline Tiago Filipe Cardoso Gouveia & $\begin{array}{l}\text { Armas, guerra y sociedad en la Edad del Hierro del noroeste } \\
\text { peninsular (el primer milenio a.C.) }\end{array}$ \\
\hline Sergio Carrasco Martínez & $\begin{array}{l}\text { Aportaciones al modelo de ocupación rural en el área central } \\
\text { de la Contestania ibérica. La Moleta (Alcoy, Alicante) }\end{array}$ \\
\hline Ana María Charquero Ballester & $\begin{array}{l}\text { La fotogrametría digital como forma de documentación } \\
\text { arqueológica }\end{array}$ \\
\hline Helena Ferrero Marín & Enterramientos de la Edad del Bronce en el País Valenciano \\
\hline Francisco Forner Cayuelas & Paisaje centuriado de Ilici (La Alcudia de Elche, Alicante) \\
\hline Ma del Carmen González Martí & La estación de La Encina (Villena, Alicante) \\
\hline Pablo Hernández Nieto & $\begin{array}{l}\text { Los Molinos de la Rambla de Puça. Propuesta de puesta en } \\
\text { valor }\end{array}$ \\
\hline María Teresa Juan Sanchís & $\begin{array}{l}\text { Maqabir en la provincia de Alicante y usos funerarios } \\
\text { islámicos }\end{array}$ \\
\hline María Lillo Bernabeu & $\begin{array}{l}\text { "Mujeres levantinas". Una aproximación al estudio del arte } \\
\text { rupestre en las tierras valencianas }\end{array}$ \\
\hline Alonso Martínez Salvador & $\begin{array}{l}\text { Evidencias arqueológicas de la minería prerromana en } \\
\text { Cartagena: la explotación minero-metalúrgica del Cabezo de } \\
\text { La Escucha en Cala Reona }\end{array}$ \\
\hline Juan José Mataix Albiñaña & $\begin{array}{l}\text { Poblamiento y territorio. La Foia de Castalla durante la } \\
\text { Prehistoria }\end{array}$ \\
\hline Alfonso Enrique Payá Pericas & $\begin{array}{l}\text { La utilización de las cuevas en la zona de L Alcoià y El Comtat } \\
\text { durante la Edad del Bronce }\end{array}$ \\
\hline Eldena María Pérez González & Arqueología y gestión del patrimonio \\
\hline Joaquín Pina Mira & $\begin{array}{l}\text { De la alquería a la pobla. Transformaciones del espacio } \\
\text { islámico con la conquista feudal en el territorio medieval de } \\
\text { Calp }\end{array}$ \\
\hline Daniel Pujalte Pérez & $\begin{array}{l}\text { Estudio de las necrópolis halladas en la partida de Almarra } \\
\text { (Castalla, Alicante). Una aproximación al entorno rural en } \\
\text { La Foia de Castalla entre los siglos X - XIII }\end{array}$ \\
\hline Alberto Ochoa García & $\begin{array}{l}\text { El proyecto meseográfico del Museo de Historia de Sax } \\
\text { (Alicante) }\end{array}$ \\
\hline
\end{tabular}




\begin{tabular}{|l|l|}
\hline Patricia Orts Escobar & $\begin{array}{l}\text { El patrimonio histórico como recurso educativo de las } \\
\text { personas con problemas de visión. Una propuesta para el } \\
\text { Tolmo de Minateda. }\end{array}$ \\
\hline Vicente J. Requena Iñiguez & $\begin{array}{l}\text { Materiales y técnicas constructivas de la villa romana de } \\
\text { L'Albir }\end{array}$ \\
\hline $\begin{array}{l}\text { Manuel Alejandro Sánchez } \\
\text { Calvo }\end{array}$ & Lloixa. Una realidad arqueológica por descubrir \\
\hline Adela Sánchez Lardíes & $\begin{array}{l}\text { Aproximación al poblamiento prehistórico en la Marina } \\
\text { Baixa (Alicante) }\end{array}$ \\
\hline
\end{tabular}

\begin{tabular}{|c|c|}
\hline \multicolumn{2}{|c|}{$\begin{array}{l}\text { IV Congreso de Máster } \\
\text { Trabajos Fin de Máster (TFM) curso 2010-11 }\end{array}$} \\
\hline Alumno/a & Contenido \\
\hline Juan Francisco Álvarez Tortosa & $\begin{array}{l}\text { Poblamiento rural y explotación agrícola en la Italia central } \\
\text { (ss. II a.n.e. - II d.n.e) }\end{array}$ \\
\hline Ana Andreu Quiles & $\begin{array}{l}\text { Aproximación al estudio de la cerámica vidriada "Melada"de } \\
\text { madînat al-zahrâ" }\end{array}$ \\
\hline Pablo Baldó López & Arqueología de la guerra civil. Línea Inmediata a Valencia \\
\hline Cristina Biete Bañón & $\begin{array}{l}\text { Estudio tecnológico y petrográfico de la cerámica del } \\
\text { yacimiento neolítico de El Prado (Jumilla, Murcia) }\end{array}$ \\
\hline Pilar Campello Payá & $\begin{array}{l}\text { Plan de puesta en valor del sistema renacentista de defensa } \\
\text { de la costa en villajoyosa }\end{array}$ \\
\hline Marina Cardillo Mata & Bodega subterránea de Fuensaldaña \\
\hline Antonio Cortés Samper & $\begin{array}{l}\text { Valoración funcional y cuantitativa del ajuar doméstico } \\
\text { ibérico en el ámbito valenciano (siglos V-I a.C.) }\end{array}$ \\
\hline Maㅡ Teresa Fernández Montoya & Hidráulica romana en el mundo rural: la Villa de Rufio (Italia) \\
\hline María Belén Jiménez Muñoz & Arqueología hidráulica del Barranc dels Molins (Ibi, Alicante) \\
\hline Iván Jover Peris & $\begin{array}{l}\text { El mundo funerario en la cuenca alta y media del río Serpis } \\
\text { durante la Antigüedad Tardía (s. IV-VII d.C.) }\end{array}$ \\
\hline Cristina Manzaneda Martín & Aportación al estudio de los santuarios oretanos \\
\hline Carlos Marco Calatayud & Estudio de la zona de El Campet (Aspe, Alicante) \\
\hline Sergio Martínez Monleón & $\begin{array}{l}\text { El Argar en el Bajo Segura y Bajo Vinalopó: aproximación al } \\
\text { modelo de ordenación y jerarquización del territorio y del } \\
\text { espacio social }\end{array}$ \\
\hline Anna Matarredona Martínez & El Molí de la Llobeta (Villajoyosa) \\
\hline Miriam Parra Villaescusa & $\begin{array}{l}\text { Dénia, clau del Regne. Formación de un espacio feudal "Ultra } \\
\text { Xuquer" (siglos XIII-XVI) }\end{array}$ \\
\hline
\end{tabular}




\begin{tabular}{|l|l|}
\hline Miriam Ruiz López & $\begin{array}{l}\text { El yacimiento arqueológico de Coimbra del Barranco Ancho } \\
\text { (Jumilla, Murcia) }\end{array}$ \\
\hline Natalia Sala Pérez & $\begin{array}{l}\text { La cerámica de uso arquitectónico en Novelda: la azulejería } \\
\text { de finales del siglo XIX y principios del XX }\end{array}$ \\
\hline Patricia Sánchez Martínez & Las termas romanas en el Sureste de Hispania \\
\hline José Manuel Torrecillas Segura & $\begin{array}{l}\text { Arqueología de un poder feudal en el Reino de Valencia: el } \\
\text { caso de los Lauria }\end{array}$ \\
\hline Aránzazu Vaquero González & $\begin{array}{l}\text { Los amuletos de la tumba número } 5 \text { de la necrópolis } \\
\text { orientalizante de les Casetes (Villajoyosa, Alicante) }\end{array}$ \\
\hline
\end{tabular}

\begin{tabular}{|c|c|}
\hline \multicolumn{2}{|c|}{ Trabajos Fin de Máster (TFM) curso 2011-12 } \\
\hline Alumno/a & Contenido \\
\hline Iván Amorós López & La Cova de l'Agüela \\
\hline Raúl Berenguer González & Análisis microespacial del Templo Ibérico de La Escuera \\
\hline María Esther Berral Sabater & $\begin{array}{l}\text { Análisis de la puesta en valor de yacimientos ibéricos de la } \\
\text { fachada mediterránea }\end{array}$ \\
\hline Álvaro Castaños Montesinos & $\begin{array}{l}\text { Organizaciones gentiliciasy sociedades de clases en la Antigua } \\
\text { Grecia (XII-VI a.N.E.) }\end{array}$ \\
\hline Héctor de Arriba González & $\begin{array}{l}\text { La Colonia de Santa Eulalia. Estudio y recuperación de un } \\
\text { espacio agroindustrial del siglo XIX }\end{array}$ \\
\hline Jessica Denia Pascual & $\begin{array}{l}\text { Lucentum, La Illeta dels Banyets y La Alcudia. Análisis y } \\
\text { comparación de las puestas en valor }\end{array}$ \\
\hline Ma Ángeles Díaz Tena & $\begin{array}{l}\text { El Bronce final y las cerámicas decoradas del Levante } \\
\text { peninsular }\end{array}$ \\
\hline Nuria Fernández Álvarez & $\begin{array}{l}\text { Propuesta de exposición temporal. Yacimiento arqueológico } \\
\text { de El Oral }\end{array}$ \\
\hline Olga Fuentes Sebastiá & Sella: paisaje y patrimonio \\
\hline Alejandro González Alegre & $\begin{array}{l}\text { La riqueza en la basura. S.I.C.E.A. (Sistema de Información, } \\
\text { Catalogación y Estudio Arqueozoológico) aplicado a un } \\
\text { estudio diacrónico de los restos de la excavación de la muralla } \\
\text { Oeste de la ciudad de Ilici. }\end{array}$ \\
\hline María Dolores Lloret Pérez & La decoración tallada del yacimiento de Xauxelles, Villajoyosa \\
\hline Noelia López Sabater & $\begin{array}{l}\text { El patrimonio histórico del Vinalopó Mitjà en la literatura de } \\
\text { Azorín }\end{array}$ \\
\hline Desiree Manzanera Quiles & $\begin{array}{l}\text { Estudio de la tumba principesca de La Escuera (San Fulgencio, } \\
\text { Alicante) }\end{array}$ \\
\hline
\end{tabular}




\begin{tabular}{|l|l|}
\hline Vicente Martí La Peña & $\begin{array}{l}\text { Topografía i empremtes arqueològiques del poblament rural } \\
\text { andalusí a l'entorn de Daniya, Dénia }\end{array}$ \\
\hline Lidia Olivo García & Defensa costera en la Sierra Gelada (ss. XVI-XVII) \\
\hline Liborio Ruiz Molina & $\begin{array}{l}\text { El yacimiento arqueológico de Los Torrejones (Yecla, } \\
\text { Murcia). Gestión patrimonial y puesta en valor }\end{array}$ \\
\hline $\begin{array}{l}\text { Celso Miguel Sánchez } \\
\text { Mondéjar }\end{array}$ & $\begin{array}{l}\text { Culto y representación de Tinnit/Démeter en el Sureste de la } \\
\text { Península Ibérica }\end{array}$ \\
\hline María Lourdes Santana Pastor & $\begin{array}{l}\text { Proyecto de reforma de las salas de arqueología del Museo } \\
\text { "Dámaso Navarro" de Petrer (Alicante) }\end{array}$ \\
\hline Susana Serra Pacheco & Relleu paso a paso. Descubriendo una cultura \\
\hline $\begin{array}{l}\text { Susana Helena Soler } \\
\text { Beresaluze }\end{array}$ & El Puntal de Salinas (Salinas, Alicante) \\
\hline
\end{tabular}

\begin{tabular}{|c|c|}
\hline \multicolumn{2}{|c|}{ Trabajos Fin de Máster (TFM) curso 2012-13 } \\
\hline Alumno/a & Contenido \\
\hline Laura Arce Cueto & $\begin{array}{l}\text { ¿Qué hace una cerámica nurágica como tú en un sitio } \\
\text { como este? Explorando las interacciones culturales en el } \\
\text { Mediterráneo centro-occidental (IX-VII a.C.) }\end{array}$ \\
\hline Almudena Barrero Osuna & $\begin{array}{l}\text { Propuesta de musealización de la necrópolis oriental de Baelo } \\
\text { Claudia (Tarifa, Cádiz) }\end{array}$ \\
\hline Rubén Cabezas Romero & $\begin{array}{l}\text { El Cabezo de la Escoba (Villena, Alicante) en los inicios de la } \\
\text { Edad del Bronce en tierras valencianas: nuevas aportaciones }\end{array}$ \\
\hline Pablo Camacho Rodríguez & Las fíbulas de los Vettones \\
\hline Rafael Chacopino Pianelo & $\begin{array}{l}30 \text { años de intervenciones arqueológicas y patrimoniales en } \\
\text { la isla de Nueva Tabarca }\end{array}$ \\
\hline Andrea Gatti & $\begin{array}{l}\text { El Arte Rupestre en la Comunidad Valenciana: modelos de } \\
\text { puesta en valor }\end{array}$ \\
\hline María Jesús Gomis Sempere & $\begin{array}{l}\text { Proyecto de puesta en valor de La Picola Portus Illicitanus } \\
\text { (Santa Pola, Alicante): Del abandono periférico a la } \\
\text { integración social y urbana }\end{array}$ \\
\hline $\begin{array}{l}\text { Máa del Rosario Gutiérrez } \\
\text { Marcos }\end{array}$ & Los oficios en la azulejería de la colección Quiles Antón \\
\hline Víctor M. Hidalgo Pinillos & $\begin{array}{l}\text { Aproximación al estudio del parcelario antiguo en la provincia } \\
\text { de Albacete: la centuriación de Libisosa }\end{array}$ \\
\hline Diana López Arroyo & $\begin{array}{l}\text { Aproximación a la reconstrucción del paisaje histórico: } \\
\text { Proyecto l'Almiserà }\end{array}$ \\
\hline
\end{tabular}




\begin{tabular}{|l|l|}
\hline Sergio Mieres Pérez, & $\begin{array}{l}\text { Aplicaciones de la prospección geofísica en España: técnicas, } \\
\text { resultados y propuestas metodológicas }\end{array}$ \\
\hline Tamara Peña Castillo & $\begin{array}{l}\text { Julio Furgús y las necrópolis de Baelo Claudia (Tarifa, Cádiz). } \\
\text { Aproximación al estudio de la exploración arqueológica de } \\
1907\end{array}$ \\
\hline Ana Peña Vizcaino & $\begin{array}{l}\text { Ilici: estudio de una ciudad romana a través de sus elementos } \\
\text { arquitectónicos }\end{array}$ \\
\hline Miguel Ángel Pérez Serrano & $\begin{array}{l}\text { El Camí del Peix (La Vila Joisosa-Alcoi): una ruta entre el mar } \\
\text { y la montaña fosilizada en la memoria }\end{array}$ \\
\hline Ma Teresa Richart Parra & $\begin{array}{l}\text { Laudas funerarias de cerámica en Biar: siglos XIX principios } \\
\text { del XX }\end{array}$ \\
\hline
\end{tabular}

\begin{tabular}{|c|c|}
\hline \multicolumn{2}{|c|}{$\begin{array}{c}\text { VII Congreso de Máster } \\
\text { Trabajos Fin de Máster (TFM) curso 2013-14 }\end{array}$} \\
\hline Alumno/a & Contenido \\
\hline Ekaterina Atrashkevich & $\begin{array}{l}\text { Muelle de mercancías de la estación La Marina: propuesta de } \\
\text { recuperación y puesta en valor }\end{array}$ \\
\hline Laura Castillo Vizcaino & $\begin{array}{l}\text { El poblamiento de época ibérica en el territorio del oppidum } \\
\text { de Peñas de San Pedro (Albacete) }\end{array}$ \\
\hline Ana Isabel Castro Carbonell & $\begin{array}{l}\text { Les Coves dels pescadors de El Campello: propuesta de } \\
\text { recuperación, puesta en valor y uso público de un patrimonio } \\
\text { olvidado }\end{array}$ \\
\hline Carlos J. del Río Villaseñor & Musealización de contextos funerarios en Museos de Alicante \\
\hline Alejandro Guzmán Villegas & Bronces romanos de la provincia de Alicante \\
\hline Iván López Salinas & $\begin{array}{l}\text { (Re)excavando El Malacón (Nerpio, Albacete): revisión } \\
\text { bibliográfica, historiográfica-documental y material para } \\
\text { una futura propuesta de estudio }\end{array}$ \\
\hline Carmen Martínez Martínez & $\begin{array}{l}\text { La villa C/Olimpo y el análisis de la periferia urbana de } \\
\text { Lucentum }\end{array}$ \\
\hline Víctor Martínez Rubio & Poblamiento rural romano en la Vega Baja del Segura \\
\hline Felipe Mejías López & $\begin{array}{l}\text { Manantiales, acequias y alquerías. Caracterización } \\
\text { arqueológica de la huerta islámica y Bajomedieval en torno } \\
\text { a un eje vertebrador del poblamiento: el río Tarafa (Aspe, } \\
\text { Alicante). }\end{array}$ \\
\hline Aitor Pérez Escobar & $\begin{array}{l}\text { El yaciment ibèric del Puig d'Alcoi: anàlisi arqueológica de les } \\
\text { excavacions Rubio (1986-1987) }\end{array}$ \\
\hline
\end{tabular}




\begin{tabular}{|l|l|}
\hline Eloy Poveda Hernández & $\begin{array}{l}\text { El poblamiento prehistórico en la Vall de Biar (Alicante): } \\
\text { nuevas aportaciones }\end{array}$ \\
\hline Pedro Juan Roselló Sendra & El Tossal del Morquí \\
\hline Ana T. Samaniego Espinosa & La formación arqueológica inclusiva \\
\hline Antonio Sánchez Verdú & $\begin{array}{l}\text { La excavación, conservación y puesta en valor de las calzadas } \\
\text { romanas) }\end{array}$ \\
\hline Katia Regina Vivas Cid & $\begin{array}{l}\text { Nabia y las ninfas en los cultos relacionados con las aguas en } \\
\text { el noroeste (siglos I-III d.C.) }\end{array}$ \\
\hline
\end{tabular}

\begin{tabular}{|c|c|}
\hline \multicolumn{2}{|c|}{ Trabajos Fin de Máster (TFM) curso 2014-15 } \\
\hline Alumno/a & Contenido \\
\hline Miriam Alba Luzón & $\begin{array}{l}\text { La vajilla campaniforme decorada de Peñón de la Zorra } \\
\text { (Villena, Alicante) }\end{array}$ \\
\hline Ana Ma Álvarez Fortes & $\begin{array}{l}\text { Ellegadode Pedrolbarra(1858-1934) enelMuseuArqueològic } \\
\text { i d'Història d'Elx Alejandro Ramos Folqués (MAHE). Los } \\
\text { materiales del Elche andalusí }\end{array}$ \\
\hline Ricardo Basso Rial & $\begin{array}{l}\text { Los procesos de especialización laboral en el sureste y levante } \\
\text { de la Península Ibérica durante la prehistoria reciente: la } \\
\text { producción textil }\end{array}$ \\
\hline Yolanda Carrasco Molina & $\begin{array}{l}\text { Proyecto de puesta en valor del Patrimonio Arqueológico de } \\
\text { época medieval islámica de Elda y Petrer }\end{array}$ \\
\hline Bernat Cucarella Pintor & $\begin{array}{l}\text { El riu Xúquer i els seus afluents com a xarxes comercials en el } \\
\text { Ferro Antic (s. VII-VI a.n.e.) }\end{array}$ \\
\hline Blanca Domínguez Martín & $\begin{array}{l}\text { Aproximación a los contextos cerámicos del oppidum ibérico } \\
\text { de La Vila Joiosa }\end{array}$ \\
\hline Leticia González Chouciño & $\begin{array}{l}\text { Proyecto de puesta en valor de los restos de la guerra civil en } \\
\text { la ciudad de Alicante }\end{array}$ \\
\hline Andrei Hindli & $\begin{array}{l}\text { Arqueología en contextos religiosos célticos en la Península } \\
\text { Ibérica: Problemas metodológicos e interpretativos }\end{array}$ \\
\hline Sergio Javier Lledó Ramírez & $\begin{array}{l}\text { Las flotas de la guarnición romana en las costas de Hispania } \\
\text { a través de las evidencias epigráficas }\end{array}$ \\
\hline Francisco José López Pando & $\begin{array}{l}\text { Análisis SIG del poblamiento Ibero-Romano del medio } \\
\text { Vinalopó }\end{array}$ \\
\hline Juan Martínez Baldero & $\begin{array}{l}\text { Aporte al estudio del urbanismo medieval oriolano a través de } \\
\text { los materiales de un solar de la calle Feria (Orihuela, Alicante) }\end{array}$ \\
\hline
\end{tabular}




\begin{tabular}{|c|c|}
\hline Miguel Martínez Baldero & $\begin{array}{l}\text { Objetos metálicos celtibéricos en La Rioja: Tipología y } \\
\text { funcionalidad, cronología, contextualización y dispersión en } \\
\text { la Comunidad Autónoma de La Rioja }\end{array}$ \\
\hline Alba Martínez Pérez & $\begin{array}{l}\text { Nuevos espacios urbanos y productivos en el Portus Ilicitanus- } \\
\text { Picola }\end{array}$ \\
\hline Jessica Mogollón Montaño & $\begin{array}{l}\text { Estudio de los objetos de metal de la Pobla Medieval de Ifach } \\
\text { (Calpe, Alicante) }\end{array}$ \\
\hline Francisco Morales Tomás & $\begin{array}{l}\text { El uso de plaquetas de sílex en el III milenio entre las cuencas } \\
\text { de los ríos Júcar y Segura }\end{array}$ \\
\hline María Pastor Quiles & La construcción con tierra en arqueología \\
\hline Zoraida Pilar Pérez Jordá & $\begin{array}{l}\text { Propuesta de guía didáctica para el museo arqueológico de } \\
\text { Santa Pola (Alicante) }\end{array}$ \\
\hline Marina Beatriz Pons Murcia & $\begin{array}{l}\text { Aproximación al estudio del yacimiento de El Moncayo } \\
\text { (Guardamar del Segura, Alicante) }\end{array}$ \\
\hline Verónica Queipo Egido & $\begin{array}{l}\text { Representación de la figura del jinete íbero en el Sureste } \\
\text { peninsular }\end{array}$ \\
\hline María Andrea Rojas Montes & $\begin{array}{l}\text { Los museos arqueológicos en Guatemala: De la gestión pública } \\
\text { a la creciente privatización }\end{array}$ \\
\hline Ivan Roldán Vergaraechea & $\begin{array}{l}\text { Arqueología de la III Guerra Carlista en el frente de Estella } \\
\text { (Navarra) }\end{array}$ \\
\hline María Jesús Quirós Ramón & Los ídolos oculados entre las cuencas de los ríos Júcar y Segura \\
\hline Rubén Santana Onrubia & $\begin{array}{l}\text { Estudio comparativo de las villas romanas en varias regiones } \\
\text { de Hispania (siglos III-V d.C.) }\end{array}$ \\
\hline Bernia Sanz Kite & $\begin{array}{l}\text { El comerç anfòric baiximperial en el vicus romà de Banys de } \\
\text { la Reina (Calp) }\end{array}$ \\
\hline Pedro José Saura Gil & $\begin{array}{l}\text { El territorio campaniforme en las cuencas del Vinalopó y } \\
\text { Serpis }\end{array}$ \\
\hline Antonio Sellés Rodríguez & $\begin{array}{l}\text { La fossa fastigata del campamento militar romano- } \\
\text { republicano de Villajoyosa: estudio de sus materiales y del } \\
\text { territorio }\end{array}$ \\
\hline Fernando Simón Oliver & $\begin{array}{l}\text { Hábitat y territorio en la cuenca del río Mundo, la Sierra del } \\
\text { Segura (Albacete) }\end{array}$ \\
\hline Octavio Torres Gomariz & Caracterizando el postalayótico balear \\
\hline Pedro Jaime Zaragozí Llopis & $\begin{array}{l}\text { El mausoleo romano de L'Albir (L'Alfàs del Pí, Alacant). Estudio, } \\
\text { interpretación y contexto }\end{array}$ \\
\hline
\end{tabular}

